\title{
Human factors in accidents *
}

\author{
M F Allnutt
}

Qual Saf Health Care 2002;11:369-375

At first sight, an article on human error based largely on military aviation accidents may appear to be inappropriate material for this journal, particularly when it is written by one whose total knowledge of anaesthesia has been confined to two sessions in a dentist's chair; but a moment's reflection may show that errors in the air and errors in the operating theatre have much in common. Thus both pilots and doctors are carefully selected highly trained professionals who are usually determined to maintain high standards, both externally and internally imposed, whilst performing difficult tasks in life-threatening environments. Both use high technology equipment and function as key members of a team of specialists, although not always with colleagues of their choosing, and are sometimes forced to operate at a time and under conditions which are far from ideal. Finally, they both exercise high level cognitive skills in a most complex domain about which much is known, but where much remains to be discovered; aeronautics, medicine, meteorology, pharmacology, etc. continue to be very active research areas.

Both pilots and doctors make many errors-that is, errors as defined by the strictest criterion of "performance which deviates from the ideal". However, the vast majority of the errors which they commit either are trivial or are easily rectified; thus an approach speed which is a knot or so too fast, or a poorly-worded communication, will probably dent only professional pride. Indeed, for all honest people, each day contains a plethora of trivial errors such as forgetting to fill the kettle, stopping at a green light, or failing to notice the duplication of a word in a sentence. Usually there is sufficient slack in the system for the error to be ignored or noticed and corrected, but some apparently innocuous errors are not noticed and some systems are not so forgiving as others; for example, a high performance aircraft or a nuclear power plant will function through a host of complex interactions and be what engineers describe as "tightly coupled" (Perrow, 1984). That is to say that what happens in one part of the system directly, and often very quickly, affects other parts. Thus recovery from a control error when flying at high speed, low level may not be possible, whereas the same error in the cruise might barely occasion comment. Therefore, for both pilot and doctor one of their frequent errors may, very occasionally, lead to a catastrophe or, in the often quoted words of Cherns, "An accident is an error with sad consequences" (Cherns, 1962).

\section{ACCIDENT CAUSATION}

Research into a number of accident areas such as aviation (McFarland, 1953; Wansbeek, 1969; Rolfe, 1972; Allnutt, 1976), nuclear power (Kemeney, 1979; Reason, 1986a) and maritime transport (Clingan, 1981; Wagenaar, 1986) shows that accidents are rarely produced by a single cause, but rather

* This is a reprint of a paper that appeared in British Journal of Anaesthesia, 1987, Volume 59, pages 856-864. by a host of interacting ones-the proverbial "chapter of accidents". Indeed some investigators believe that looking for the cause of an accident is damaging (Holladay, 1973), while some organizations choose to distinguish between "primary" and "secondary" causes and some between "necessary" and "sufficient" ones (Wagenaar, 1986). What is clear is that, whatever the categories used, many accidents are ascribed to "human error". In general aviation this figure varies between 42 and $87 \%$ (Feggetter, 1985), the figure tending to be higher for the simpler systems such as light aircraft. The term "human error", however, is often only a synonym for "pilot error", although the pilot may in fact be taking the blame for the real guilty party - the manager, trainer, aircraft designer or ground controller. So human error in the operating theatre might be the fault of the anaesthetist, but it might equally well be the fault of the person who failed to train him or her correctly or the person who failed to pass on a message, or who designed, bought, or authorized the purchase of, an inadequate piece of equipment. There is an extreme view which argues that all accidents result from human error and that those which we ascribe to "technical failure" or "act of God" merely reflect our ignorance or unwillingness to probe sufficiently deeply. However, for practical purposes let us merely say that accidents are usually complex and are caused, or at least exacerbated by, many factors and that human error often plays a large part in their causation.

An absolutely basic tenet of this paper is that all human beings, without any exception whatsoever, make errors and that such errors are a completely normal and necessary part of human cognitive function. For a pilot or doctor to accept that he or she is as likely as anyone else to make a catastrophic error today is the first step towards prevention; whereas to claim exemption on the grounds of being a test pilot, senior professor, commanding officer or consultant, or of having 30 years' experience or 3000 accident-free hours, is the first step on the road to disaster.

As hindsight is normally far superior to either foresight or insight, the most common starting point for a treatise on human error is the accident and its sequelae. This will lead us into a consideration of normal cognitive function and the operator's interaction with his or her colleagues (communication errors), equipment (machine-aided errors) and environment (environment-aided errors). While the example quoted will be taken mainly from military aviation, the underlying mechanisms apply equally to cockpit and operating theatre and the reader is invited to supply his or her own examples. (Reports on individual military accidents have a restricted circulation and consequently are not cited in this paper.)

\section{ACCIDENT INVESTIGATION}

From the moment that an aircraft crashes, or an accident occurs in the operating theatre, one of the two major sources of evidence starts to decay rapidly and to become distorted. This source is the memory of the participants, both direct and indirect, for the event. A large amount of laboratory and anecdotal evidence show that memory decreases rapidly over 
time and that it is distorted in the direction of simplicity and coherence (Bartlett, 1932; Baddeley, 1976). We seem to hate informational chaos and to have a basic need to structure a situation and to provide a coherent account of what happened (Bartlett's "effort after meaning"). Thus, with the highest integrity, we soon start reporting not what happened, but what must have happened. Incidentally, the other major source of post-accident evidence (the physical factors such as the state of the aircraft engine, marks on the ground, documentation etc) may or may not also be subject to decay, but this is outside the remit of this paper.

The primary tasks after an accident are of course to prevent further damage and to look after the injured; but second only to these is the need to facilitate the inquiry by minimizing the decay and distortions of memory (Allnutt, 1973). Thus all those who are involved in any way with the accident are encouraged to write down open-ended statements as soon as possible after the event (the statements are then impounded) and are advised, often successfully, to refrain from discussing the accident until interviewed by the professional investigators.

Military aviation accidents, in common with almost every other type of accident, are always a surprise and invariably occur at the most inopportune time and place. Nevertheless, a team consisting of two pilots, an engineer and a psychologist (plus other specialists if necessary) will try to get to the scene with all speed. They carry with them their luggage and their preconceptions; for as soon as the barest details of an accident are broadcast the cognoscenti immediately "know" what (must have) happened. ("That always was a dangerous manoeuvre"/"I always said young Jones was an accident waiting to happen".) Even investigators will have their prejudices and theories; they may be overly sympathetic towards a pilot and they will make mistakes (even the psychologist!). In short, they will further distort the picture of what really happened.

The purpose of an accident investigation is to ascertain what happened and why it happened, so that systems and procedures can be improved (Rouse and Rouse, 1983), rather than to apportion blame, which may be dealt with by a subsequent inquiry. Such is the intent; but by their very nature accident inquiries are emotional occasions because families have been devastated, careers wrecked, etc. Investigators are human, and most would be happiest if the cause of the accident could be shown to be technical failure (human error at a distance) or freak weather conditions, but often there patently has been human error and the investigator may be exposed to the whole gamut of distortions of the truth ranging from the bare-faced lie to save one's own or a colleague's skin, through repression, to the witness being just a shade too selective in his account of events or letting a false nuance pass unchallenged.

The investigator must try to tease out the truth while taking great care not to lead the witness. An example of the care needed in the phrasing of questions is shown by Loftus's study in which subjects viewed a video film of a car crash and were then asked to estimate the car's speed at impact. The question asked of four matched groups of subjects was identical except for the verb used which was either "contacted", " hit", "collided" or "smashed". The subsequent estimates of speed correlated positively with the "violence" of the verb, ranging from 31 to $41 \mathrm{mph}$ (Loftus and Palmer, 1974). Other problems with interviewer bias (Schmitt, 1976) and eye-witnesses (Wells and Loftus, 1983) must also be considered. At the end of this analysis the investigator may conclude that one of the factors which caused or exacerbated the accident was human error.

\section{COGNITIVE FUNCTION}

The pilot of a modern military aircraft is bombarded by a plethora of information from his instruments, environment, co-pilot, ground control, etc. He can only hope to process a small part of this input and his skill lies in simplifying the complex task by dealing correctly with the critical information at just the correct time. When he fails to do this an error, and possibly an accident, may occur. Although there is much current research and debate in psychology on some of the finer points of cognitive function, there is fairly widespread agreement on the basics of the system, as described for example by Wickens (1984) and Sanford (1985). First, the stimulus must fall within the range of the pilot's senses - that is, only a fairly narrow band of sound can be sensed and velocity can only be inferred. After sensation comes perception, for a stimulus does not fall onto a tabula rasa but rather onto a very active mind, and is rapidly converted into a meaningful percept. We then attend selectively to only a few of these percepts; the degree of attention varies and there is evidence to show that we process incoming stimuli to a variety of levels depending on many factors (Craik and Lockhart, 1972), but a simple binary division will suffice for this discussion of human error.

This simple division is between low-level processing where we appear to process very large amounts of information easily, very rapidly and in parallel, and higher-level processing which is the subject of conscious attention and in which we process information sequentially and comparatively slowly. The latter is William James' "window of consciousness" (James, 1890) and only a very small but most important part of our cognitive processing uses this mechanism. Failure of the low-level mechanism gives rise to slips and failure of the higher-level one to mistakes. We shall return to these two types of error shortly. Meanwhile, our cognitive processing system is completed by two main types of memory: a shortterm "scratch-pad" where information decays in a few seconds and a long-term memory the contents of which will be distorted by both previous and subsequent events. (See Baddeley (1976) for a description of these and other types of memory.) Percepts and memories are then compared and decisions communicated to the effector mechanisms such as body movement and speech. Finally, feedback loops complete this very crude description of a hugely complex and highly sophisticated system.

\section{TWO TYPES OF ERROR}

The distinction between the two main types of error, slips and mistakes (Norman, 1980), is based on the failure of one or other of the two main processing mechanisms, fast low-level "schematic" parallel processing, or the slower high level "attentional" sequential processing. We might note that some researchers (Rasmussen, 1981; Reason, 1986a) advocate a three-category system for errors based on failures of skill-based, rule-based and knowledge-based behaviour, but a binary split appears adequate for most purposes.

In order to cope with vast amounts of incoming information, and having only a relatively slow "attentional" processing mechanism, human beings have developed and built up a very large repertoire of "schemata". These are small routines (perhaps "sub-routine" would be an appropriate computer analogy) which are called into play by very specific stimuli and require minimal conscious monitoring. These schemata are accompanied by heuristics ("rules of thumb") based on what has worked well in the past and such mechanisms are essential if we are to function in this world of information overload. Thus our daily routine for getting up, washed, dressed, fed and to the office consists of a string of over-learned schemata to which we attend closely only when something disrupts our routine. When we are not paying sufficient attention an inappropriate schema may be "captured" or called into play and a slip occurs (Reason, 1986b). For example, a tired pilot landed on a hot day and intended to 
pull a lever to open the cockpit while he taxied across the airfield. At that moment he was distracted by a radio call which took his attention and he slipped into his post-take-off routine, pulling an adjacent lever which raised the undercarriage or (lowered the aircraft, as the pedants will insist). He realized his slip just too late and was heard to mutter the Naval synonym for "Oh bother"!

Diary studies (Reason and Mycielska, 1982) show how slips pervade everyday behaviour and are most likely to occur during the performance of highly automated tasks in familiar surroundings when attention is elsewhere because of boredom, preoccupation, or distraction. In particular, errors of omission are often caused by unexpected interruptions which may cause us to go back to a behavioural sequence at the wrong place. These slips are not arbitrary but tend to take fairly predictable forms (Norman, 1981). "When cognitive operations are under-specified they tend to default to contextually appropriate high frequency responses" (Reason, 1986b). This, as Reason acknowledges, is a derivation from the message of earlier authors such as the "false hypothesis" (Davis, 1958) and "response bias" (Broadbent, 1967). In short, when we don't have quite enough information we tend to go for what has worked in the past and we "see" what we expect to see and "hear" what we expect to hear.

A good demonstration of the tendency to default to a highfrequency response (or "frequency gambling") is the phonological priming game played by children. In this someone might be asked a rapid series of questions which provoke answers such as "most", "boast", "host", etc, and is then asked what is put into a toaster; he or she often defaults to "toast". As our expertise in a particular area increases, so does the problem of slips, for one way of defining an expert is someone who has built up a vast repertoire of appropriate and finely graded schemata which allows him or her to carry out many very complex procedures while devoting much of his or her attention to the "bigger issues". However, the penalty for this expertise is that the more schemata we possess, particularly subtle gradations of appropriate response, the more likely it is that the wrong schemata will be called into play when attention is elsewhere; that is to say, experts are, in general, more likely than novices to make slips. Some comfort may be drawn from Woods (1984) who showed that slips are detected far more often than mistakes.

Whilst slips are errors in which the intended action was correct but the actual action wrong, the second category of error, the mistake, is where the intention itself was wrong. Laboratory research and real-life observation indicate that human decision-making is often far from ideal and that we excel as pattern recognizers but not as calculators (Tversky and Kahneman, 1974). "Humans if given the choice would prefer to act as context-specific pattern recognizers rather than attempting to calculate or optimize" (Rouse, 1982). Having recognized the pattern or problem as analogous to one we have faced before, we quickly provide the first hypothesis which comes to mind and tend to stick with it, or "first come best preferred" (Reason, 1986a). If the hypothesis is correct, as it usually is, we enhance our reputation for decisiveness, but when it isn't we will often be very slow to change it. Again, both laboratory and observational evidence indicate that we prefer to seek confirmatory evidence rather than putting our hypothesis to a real test. The military accident literature contains many examples of pilots demonstrating this "confirmation bias" by making a navigational error and then "interpreting" a great deal of subsequent information to support their initial (erroneous) hypothesis. Similarly, as a slow-moving emergency develops, operators tend to take a "keyhole" approach, making a quick initial hypothesis and then jumping almost randomly from one focus of concern to another in an attempt to verify it (Reason, 1986c).

\section{COMMUNICATION ERRORS}

Many errors involve the team rather than the individual and, while monitoring another's performance may sometimes prevent errors from becoming accidents, the presence of others can provide a dangerous illusion of security as "divided responsibility is no responsibility". For example, an aircraft crashed into the Everglades while all three crew members were trying to solve a minor problem; and a British airways 747 approached Nairobi airport for several minutes with none of the three crew reacting to the fact that the safety altitude had been set to 327 feet below ground level! (Aircraft Accident Report, 1975). Finally, an error may occur in the communication itself. The assumption is that as communication is about the same objective events it should be accurate, but communication is based not on the real objective world, but on our individual mental model of the world and the sender's and receiver's models may well differ significantly. Hence the much quoted, and probably apocryphal, accident caused by the pilot saying "feather four" meaning, on his understanding of the situation, "feather Number 4 engine" which was interpreted by the co-pilot, using his mental model of the situation, as "feather all four engines". History is replete with major communication blunders such as the Charge of the Light Brigade (see Reason and Mycielska (1982) for an interesting analysis of the errors), but a quick review of any day's conversations will show how people are "working from different maps". Nor must the importance of non-verbal communication be forgotten: mis-reading a gesture is a most common error.

\section{MACHINE-AIDED ERRORS}

Well-designed equipment can prevent or at least ameliorate the effects of an error, whereas poorly designed equipment is often cited as the "cause" of an accident. What the pilot or doctor requires from his instruments is clear, concise, reliable, unambiguous information to the accuracy (but no more) which he needs; the controls must be comfortable, precise, easy to operate, unambiguous and give him immediate and adequate feedback that his intended action has been effected. To minimize errors and fatigue, displays and controls must be easy to use (by the pilot) while wearing full flying clothing and under the most severe environmental conditions. Not only must each display and control meet the design criteria, but they must be logically grouped together by function and information flow, with the most important being placed in the centre of vision. They must also obey the motion stereotypes (McCormick and Sanders, 1982); that is, we have all developed the schema that turning something clockwise increases it, while switches are down for "on" (opposite schemata in the USA). Above all, the system must be designed as far as practicable to save the operator from his own errors, for Murphy stalks the airways and the maintenance hanger (and, I suspect, the operating theatre). A \$325 million Bl bomber was lost because the system allowed the pilot to cancel a warning signal without taking appropriate action (Cordes, 1985). Similarly, the gearbox of a military helicopter failed in flight because a maintenance technician read in the manual that "the thrust faces are mounted facing outwards": he assumed that this meant outwards from the engine whereas the writer meant outwards from each other. Thus an accident may well be the result of human error, but one committed many years before the event.

The advice given to a pilot who experiences a conflict between his body senses and his instruments is, "believe your instruments". This is almost invariably sound advice, but the problem comes when an instrument is known to be "sick" or when the information which it gives just cannot be fitted into any hypothesis which the operator can countenance. At one time during the Three Mile Island accident, the operators in 
the control room were faced with 114 simultaneous warnings and could not postulate an hypothesis to fit the data (Perrow, 1984). Ideally, an instrument should indicate if it is malfunctioning and the operator should have adequate procedures for continuing without it. This leads to the problem of reversionary mode procedures, for the more reliable the equipment the more "rusty" the back-up procedures and skills. The judgement about which reversionary mode procedures to train and maintain is a fine one, for there are some military flying procedures in which more people have crashed practising the reversionary procedure than following failure of the equipment itself.

There is now a strong case for arguing that there is no excuse whatsoever for poorly designed equipment and procedures in an environment in which human life is at stake. The principles of good ergonomic design have been known for several decades and are to be found in standard textbooks (Van Cott and Kincade, 1972; McCormick and Sanders, 1982). The most complex interface is between man and machine and this should be at the very centre of the design process. Various reasons are offered for poor design, such as ignorance of ergonomics, the belief that a good-looking (cosmetic) machine sells better than a safe one, or the fact that the equipment wasn't designed originally for the purpose for which it is now being used-for example, military surplus equipment is often installed in light aircraft on the grounds of cheapness, but unpressurized aircraft do not require altimeters which read to 99999 feet (Dale, 1985). Of course, the man-machine interface of the future will increasingly be a man-software rather than a man-hardware one, while the major focus of engineering psychology will continue to move from sensory motor concerns to cognitive ones (DeGreen, 1980). Although guidelines for the design of the man-computer interface exist (Smith and Aucella, 1982), they are often ignored and there sometimes appears to be an alarming trend back towards poor ergonomics; anyone who has sat in front of an expensive "user-hostile" computer which incessantly repeats "input error" will appreciate the concern.

\section{ENVIRONMENT-AIDED ERRORS}

The role of stress in accident causation is a most complex one which usually leaves us at the end of the investigation with strong suspicion rather than proof. Consider, for example, a civil aviation accident in which a pilot was kept awake for most of one night because of a domestic dispute and then, after a 12-hour duty day, was faced with an engine failure just after take-off. The inquiry team opined that fatigue may well have influenced his decision to close down the good engine rather than the one which was malfunctioning; suspicion but not proof (CAP, 1969). Although there is not even an agreed definition of stress (authors talk about "stressors", "strain", etc), the topic has spawned a vast literature of laboratory, field, observational and historical studies. The stresses themselves may be conveniently, although arbitrarily, divided into environmental stresses such as heat, noise, vibration etc, physiological ones such as sleep loss, circadian rhythms, drugs, etc., and psychological ones such as fear, frustration, competition, etc. The literature has been comprehensively reviewed by, amongst many others, Appley and Trumbull (1967) and Poulton (1971).

Of the many things which may be said about stress and human error, six simplistic generalizations may suffice. These are:

(a) Most research has been into the effects of single stresses, whilst real-life environments contain many stresses, the effects of which depend on a host of intervening variables such as the fitness, training and motivation of the operator, the nature and complexity of the task and the strength, duration, interactions and suddenness of onset of the stresses involved. (b) Objective and subjective reactions to stress are often not well correlated. Thus the real danger of alcohol is not that it degrades performance, but that it does so whilst we think our performance is good (Cohen, 1960). A related problem with alcohol is that pilots do not appreciate the duration of its effects and often feel safe just because it is a new day (Green, 1983). It is also impossible to divorce domestic from work stresses, and domestic stress has been shown to increase proneness to accidents (Rahe, 1969; Alkov, Borowsky and Gaynor, 1983). Thus a real menace is the very senior man who claims to "leave my worries behind me when I enter the cockpit" or to have "trained myself to do without sleep". He has not, but his juniors may be just a shade reluctant to point out to him the relevant psychological literature!

(c) The breakdown of performance under stress may take many forms, such as increased errors and irritability or decreased speed and accuracy, or both. Two mechanisms seem to feature in accident investigations with particular frequency. One is what is commonly referred to as "coning of attention" - that is, at a time when we most need to gather a broad spectrum of data in order to make a good decision, we concentrate on one single source, the "first come, best preferred" solution described earlier. An extreme manifestation of this phenomenon is when passengers in a crashed aircraft struggle to open a door while ignoring a large hole in the fuselage a few feet away. The second mechanism is "reversion under stress". Man cannot make himself forget and both laboratory and observational evidence shows that, under stress, recently-learned behaviour patterns may be replaced by older, better-learned ones (Fisher, 1984). Thus a small explosion on landing caused a military aircraft to veer off a runway and down a hill with the pilot furiously trying to stop it with the hand-brake. Sadly, that type of aircraft was not fitted with a hand-brake, but the aircraft which the pilot had flown for the previous 6 years had been so fitted.

(d) In general, performance seems to follow an inverted U-curve, being best at moderate levels of arousal (Yerkes and Dodson, 1908) or in the middle of the "day-dream to panic" continuum (Lager, 1973). This produces two danger points: boredom and panic. In the former, arousal is very low, attention elsewhere, and slips occur; in the latter, the "chapter of accidents" builds up inexorably until the pilot is overwhelmed and mistakes (and slips) occur.

(e) Manhood and safety may appear to be incompatible. Our culture, particularly in activities such as flying and sport, endorses concepts such as "manhood" and "pressonitis" and looks a little askance at an over-concern with safety (Mason, 1972); but this same phenomenon also appears in a much more subtle form and among many groups of professionals there is often a marked reluctance to "lose face" by admitting ignorance or fatigue and handing over to a colleague who may be more able, less fatigued and, perhaps, junior.

(f) Money and safety often appear to be pulling in opposite directions. A pilot is not employed "to fly safely", but "to fly, safely". He is there to win the war or to make a profit for his company and to do so safely. Thus safety would be enhanced enormously by not flying in bad weather, but profits would suffer and so the whole question becomes one of "acceptable risk"; for example, 31 of the 40 pilots who first flew the US Mail died in flying accidents (Perrow, 1984). It must be remembered that we are talking here about both "perceived risk" and "actuarial risk". The helicopter pilot who crashed in the mountains trying to rescue what he believed to be a badly injured man based his decision on his assessment of the risk; that the man was not in fact badly injured is irrelevant to our judgement of his decision.

\section{FUTURE ERRORS}

As our knowledge of human cognitive processes gradually improves we see that man's cognitive capacity is the fixed 
element in the system and that slips and mistakes are natural, ubiquitous behaviour occasioned by various combinations of cognitive, social and situational factors. Although we cannot predict when and where the next accident will occur, we can predict with confidence that human error accidents will continue unabated and indicate situations in which they will be more likely to occur. The key issue is whether the number of such accidents can be reduced in the future.

In some senses the situation is steadily deteriorating as man is called upon to undertake ever more challenging tasks and the potential consequences of human error in highly sophisticated, tightly-coupled systems, such as high speed aircraft and nuclear power stations (such as Chernobyl), are catastrophic. However, there are at least three reasons for optimism. The first is that much of the information necessary for the design of good "man-compatible" equipment and procedures is readily available; the missing element is the willingness to apply it. Second, while it may sound like a pious hope to expect that a better understanding of human attitudes and behaviour, particularly in a crisis, may diminish the chances of an accident, there are some encouraging signs in this direction. Many military organizations are now adopting a much more sensible attitude towards human error and are investigating ways of identifying pilots who are more likely to have accidents; various agencies, including some armed forces, are starting to use relaxation training to counter stress and one of the recommendations of the Three Mile Island enquiry was that an incident monitor should sit quietly in an isolated room away from the troubleshooting team and think about the incident as a whole (Perrow, 1984).

The third reason for optimism could in the end prove to be the most rewarding. This is the hope that a very real symbiosis between man and machine could allow each to counter the other's weaknesses. Such a productive partnership may seem very far off to those of us whose "partnership" with a personal computer seems to produce an even greater mess than we might have achieved single-handed, but the potential is there because man possesses attributes such as drive, intuition and pattern-matching skills etc, while machines are dispassionate, possess reliable and virtually unlimited memory and never tire of repetition. Work on Expert Systems, while proceeding in more of an evolutionary rather than the revolutionary manner which some had expected, is progressing. In particular, Intelligent Decision Aids (Rouse and Rouse, 1983) will be, and indeed are beginning to be, programmed to respond to the particular needs of the individual operator and to "understand" how he functions. They will collate a plethora of incoming information, make inferences and offer a decision to the operator together with information about the basis on which it was made and the probability of its being correct.

Technology marches on apace while human cognitive processes and capacity have remained remarkably stable over the centuries. Systems and procedures designed around a thorough knowledge of human cognition and attitudes have the potential to prevent, or ameliorate, some of the errors which are a normal and necessary part of human functioning. There are some encouraging signs that our attitude towards human error is improving, but it is likely to be some considerable timee before the operator, be he pilot or doctor, ceases to be the first choice for "guilty party"; hence the definition of a pilot as "the person who attends the accident".

\section{Author's affiliation}

M F Allnut, Army Personnel Research Establishment, Farnborough, Hants, UK

\section{REFERENCES}

Aircraft Accident Report (1975). Report on the incident near Nairobi Airport Kenya on September 1974. Aircraft Accident Report 14/75. London: HMSÓ
Alkov RA, Borowsky MS, Gaynor JA (1983). Pilot error and stress management. US Navy Experience. Proceedings of the IALPA Conference, Dublin

Allnutt MF (1973). The psychologist's role in aircraft accident investigation. In: Corkindale KGG, ed. Behavioural aspects of aircraft accidents. AGARD Conference Proceeding No. 132.

Allnutt MF (1976). Human factors. In: Hurst R, ed. Pilot error. London: Crosby Lockwood Staples.

Appley MH, Trumbull R (1967). Psychological stress. New York: Appleton Century-Crofts.

Baddeley A (1976). The psychology of memory. New York: Harper and Row.

Bartlett F (1932). Remembering. Cambridge: Cambridge University Press.

Broadbent DE (1967). Word-frequency effect and response bias. Psychol Rev 74: 1

CAP (1969). Report of the Italian Commission of Inquiry into the Accident to BAC 1-11 G-ASJJ at S. Donato, Milanese. London: HMSO.

Cherns AB (1962). In: Welford T, ed. Society: Problems and methods of study. London: Routledge and Keegan Paul.

Clingan IC (1981). Safety at sea. Interdisciplinary Science Reviews 6:36.

Cohen J (1960). Chance, skill and luck. Harmondsworth: Penguin Books.

Cordes C (1985). Military waste: the human factor. Am Psychol Assoc Monitor 16: 15.

Craik FIM, Lockhart RS (1972). Levels of processing: a framework for memory research. J Verbal Learning Verbal Behav 11: 671

Dale HCA (1985). The state of the office: ergonomics in general aviation-an illustrated example. In: Hurst R, Hurst L, eds. Fly and survive. London: Collins.

Davis RD (1958). Human engineering in transportation accidents. Ergonomics 2: 24

DeGreen KB (1980). Major conceptual problems in the systems management of human factors/ergonomics research. Ergonomics 23: 3

Feggetter AJF (1985). Human factors: the key to survival. In: Hurst R, Hurst L, eds. Fly and survive. London: Collins.

Fisher S (1989). Stress and the perception of control. Hillsdale, New Jersey: Lawrence Erlbaum Associates.

Green RG (1983). Alcohol and flying. Int J Av Safety 1: 23.

Holladay DH (1973). Planning to prevent the preventable. Presentation to the Seminar on Aviation Accident Prevention, Royal Institute of Technology, Stockholm, Sweden.

James W (1890). Principles of psychology. New York: Holt, Rinehart and Winston.

Kemeney J (1979). The need for change: the legacy of TMI. Report of the President's Commission on the Accident at Three Mile Island. Washington: Government Printing Office.

Lager CG (1973). Human factors. Paper presented to a Seminar on Aviation Accident Prevention, Royal Institute of Technology, Stockholm, Sweden.

Loftus E, Palmer J (1974). Reconstruction of automobile destruction: an example of the interaction between language and memory. J Verbal Learning Verbal Behav 13: 585.

McCormick EJ, Sanders MS (1982). Human factors in engineering and design. New York: McGraw-Hill.

McFarland RA (1953). Human factors in air transportation. New York: McGraw-Hill.

Mason CD (1972). Manhood versus safety. Paper presented to the Third Oriental Airlines Association Flight and Safety Seminar, Singapore.

Norman DA (1980). Errors in human performance. Center for Human Information Processing. University of California Report 8004

Norman DA (1981). Categorisation of action slips. Psychol Rev 88: 1

Perrow C (1984). Normal accidents. New York: Basic Books Inc.

Poulton EC (1971). Environment and human efficiency. Illinois: Charles C Thomas.

Rahe RH (1969). Crisis and health change. Naval Medical Neuropsychiatric Unit Report 67-4. San Diego, USA

Rasmussen J (1981). Models of mental strategies in process plant diagnosis. In: Rasmussen J, Rouse W, eds. Human detection and diagnois of system failures. New York: Plenum.

Reason JT (1986a). Recurrent errors in process environments: some implications for the design of intelligent decision support systems. In: Hollnagel E, ed. Intelligent decision support in process environments. Berlin: Springer-Verlag.

Reason JT (1986b). Cognitive under-specification: its varieties and consequences. In: Baam B, ed. The psychology of error: a window on the mind. New York: Plenum.

Reason JT (1986c). An interactionist view of system pathology. NATO Advanced Research Workshop on Failure Analysis of Information Systems, Bad Windsheim, Germany.

Reason JT, Mycielska R (1982). Absent minded? Englewood Cliffs, NJ: Prentice-Hall Inc.

Rolfe JM (1972). Ergonomics and air safety. Appl Ergonomics 3: 75.

Rouse WB (1982). Models of human problem solving: detection, diagnosis, and compensation for system failures. Proceedings of IFAC Conference of Analysis Design and Evaluation of Man-Machine Systems, Baden-Baden, Germany.

Rouse WB, Rouse SH (1983). Analysis and classification of human error. IEEE Trans Syst Man Cybern 13: 539

Sanford A (1985). Cognition and cognitive psychology. London: Weidenfeld and Nicholson.

Schmitt N (1976). Social and situational determinants of interview decisions. Personnel Psychol 29: 79. 
Smith S, Aucella A (1962). Design guideline for the user interface or computer-based information systems. Bedford, Mass: Mitre Corporation.

Tversky A, Kahneman D (1974). Judgements under uncertainty: heuristics and biases. Science 185: 1124

Van Cott HP, Kincade RG, eds (1972). Human engineering guide to system design. Washington:US Government Printing Office.

Wagenaar WA (1986). The cause of impossible accidents. The Sixth Duiker Lecture, University of Amsterdam.

Wansbeek GC (1969). Human factors in airline incidents. Paper presented to the 22nd Annual International Air Safety Seminar at Montreux.

Wells GL, Loftus EF (1983). Eyewitness testimony. Psychological perspectives. New York: Cambridge University Press.

Wickens CD (1984). Engineering psychology and human performance. Columbus, Ohio: Charles E Merritt.

Woods DD (1984). Some results on operator performance in emergency events. Institute of Chemical Engineers Symposium Series 90: 21

Yerkes RM, Dodson JD (1908). The relation of strength of stimulus to rapidity of habit-formation. J Comp Neurol Psychol 18: 459.

\section{COMMENTARY}

\section{DIAGNOSING DOCTORS}

The paper by Dr Martin Allnutt, a military aviation psychologist, is now 15 years old. ${ }^{1}$ That it was published in a mainstream anaesthesia journal that long ago reflects credit on the then Editor of the journal and his staff. For, so far as medicine was (and, regrettably, still is to a degree) concerned, this paper remains ahead of its time. Yet Dr Allnutt would be the first to point out that most of what he says could be regarded by psychologists as mainstream knowledge. ${ }^{2}$

There are so many concepts and messages in this paper that strike at the heart of error production and which are fundamental to patient safety improvements, that it must be regarded as required reading-by which I mean "understanding and acknowledging" — for all medical practitioners.

Perhaps the most powerful statement in this power packed paper is that it is "an absolutely basic tenet ... that all human beings, without any exception whatsoever, make errors and that such errors are a completely normal and necessary part of human cognitive function. For a pilot or doctor to accept that he or she is as likely as anyone else to make a catastrophic error today is the first step towards prevention; whereas to claim exemption on the grounds of being a test pilot, senior professor, commanding officer or consultant, or of having 30 years' experience or 3000 accident-free hours, is the first step on the road to disaster."

Is there a single medical practitioner who can deny the penetrating accuracy of that statement? It compels the abandonment of the often unspoken but absurd precept still fluttering about inside medicine that "doctors never make mistakes", and demands an understanding that errors are essential learning processes for all human beings. The paper pointed clearly to the need for a culture change in medicine which we are still struggling to achieve. Put simply, to deny one's errors is both ridiculous and dangerous.

Why is it that medicine persisted in denying its fallibility in the face of irrefutable, psychological and clinical (not to mention common sense) evidence to the contrary? The reasons are neither complicated nor mysterious; they are simple, human ones.

There is an eloquent Chinse proverb which says:

Victory has many fathers: defeat is an orphan!

Doctors, like all other human beings, have been reluctant to admit they were wrong. Now let us be fair. Medicine has achieved much and will continue to do so. After all, medical practitioners have obviously quite often been right! However wrong we are at times, in keeping with the rest of the human race and, as Allnutt explains, such errors are fundamental to progress.
Historically, the profession has occupied a privileged place in communities. That age old "mystery" surrounding healing (now added to in modern times by many equally "mysterious" technological advances), the relative scarcity of a university education, and the vulnerable position in which bad health places anyone led patients over the centuries to place in us their complete trust and respect. Sadly, albeit uncommonly, this trust has been abused. Like all fallible humans, we let this privileged position go to our heads. It resulted in some of us-present and past-adopting a patronising posture where admission of ignorance or error was unthinkable. This "culture" began to be perpetuated, particularly among some senior members of the profession, and even influenced the attitudes of medical undergraduates. Absurdly, the patient was pushed into the background while we paraded around in a flood of self-importance, competing for attention and seniority. The poor patients became frightened even to question us about their own health and our decisions concerning their welfare. As for being honest and open with patients and relatives about our errors, "advice" from our medical insurance and our vanity made this uncommon.

But improvements are discernible. Importantly, patients are no longer willing to sit "dumb and accepting" of our pontifications. Society has woken up to our fallibility and, happily, it demands the truth from us now. To be fair, this emerging change of attitude has received some of its impetus from within the profession itself. ${ }^{3-5}$ The cynic may say that the heat of medicolegal scrutiny initiated this, but there are-and always were-good and honest medical practitioners. An attitude of openness and honesty with patients, relatives, and colleagues is now widely practised and advised.

Of course the present tort system in the courts, with its focus on outcome and the need to assign "fault" (often of an individual), tends to fly in the face of such advice. ${ }^{6}$ Having made an error, often while subject to complex and stressful demands imposed by a less than perfect system, ${ }^{7}$ and while actually trying to do our best, medical and nursing personnel have been encouraged to "admit nothing" by our medical insurance institutions and then are subject to a legal process that operates in this "blame" culture. As mentioned above, a desire not to lose face will encourage such behaviour. This approach drives error admission and analysis underground, so that methods of preventing a recurrence are never considered. It also carries the potential for injustice, where an individual who forms the final pathway for an accident, thrust there by a flawed environmental system, takes the blame for slips and mistakes (for example, judgmental errors) made hours, days, or months earlier by other persons on the blunt managerial end. Furthermore, it is an ironical fact that keeping harmed patients and their relatives uninformed about an error mitigates powerfully towards their seeking legal redress.

Emphasising the rapid decay and complete unreliability of human memory over time-particularly when stressed-the paper firmly supports the concept of early and full reporting of incidents, near misses, and adverse events by those directly involved. Such incident reporting data, correctly amassed and analysed, are already making powerful contributions to patient safety improvements. Dr Allnutt's implied support for this technique is hardly surprising, remembering that the original concept of "critical incident reporting" appeared 30 years earlier in a seminal paper by a pioneer military aviation psychologist. $^{8}$

The insight into human cognitive processes provided by Dr Allnutt's paper is fundamental to error prevention. Its messages should become part of medical education curricula at all levels of learning. Yet appreciable unawareness of these concepts persists among many medical practitionerspersons who could reasonably have been expected to be among the most informed in such matters. Dr Allnutt is telling us not only that "patterns of human error are identifiable, 
predictable, repetitive and lend themselves to classification and analysis", ${ }^{1}$ but that they offer the very information that is the pathway to their prevention.

This is a classic paper for all interested in improving the safety of our patients, our colleagues, and ourselves.

\section{J Williamson}

Specialist Consultant, The Australian Patient Safety Foundation, 27 McKenna Street, Kensington Park, South Australia 5068; John.Williamson@apsf.net.au

\section{REFERENCES}

1 Allnutt MF. Human factors in accidents. Br J Anaesth 1987;59:856-64

2 Reason J. Human error. Cambridge: Cambridge University Press, 1990.

Senders J, Moray N. Human error: cause, prediction, and reduction.

Hillsdale, NJ: Lawrence Erlbaum Associates, 1994.

4 Bogner MS. Human error in medicine. Hillsdale, NJ: Lawrence Erlbaum Associates, 1994

5 Almaberti R. Organizational structures and safety systems. Patient Safety and Medical Error. Salzburg Seminar, Session 386, 2001.

6 Runciman WB, Merry AF, Tito F. Error, blame and the law in health care: an Antipodean perspective. Qual Saf Health Care (in press).

7 Runciman WB, Webb RK, Lee R, et al. System failure: an analysis of 2000 incident reports. Anaesth Intensive Care 1993;21:684-95.

8 Flanagan JC. The critical incident technique. Psychol Bull $1954 ; 51: 327-58$

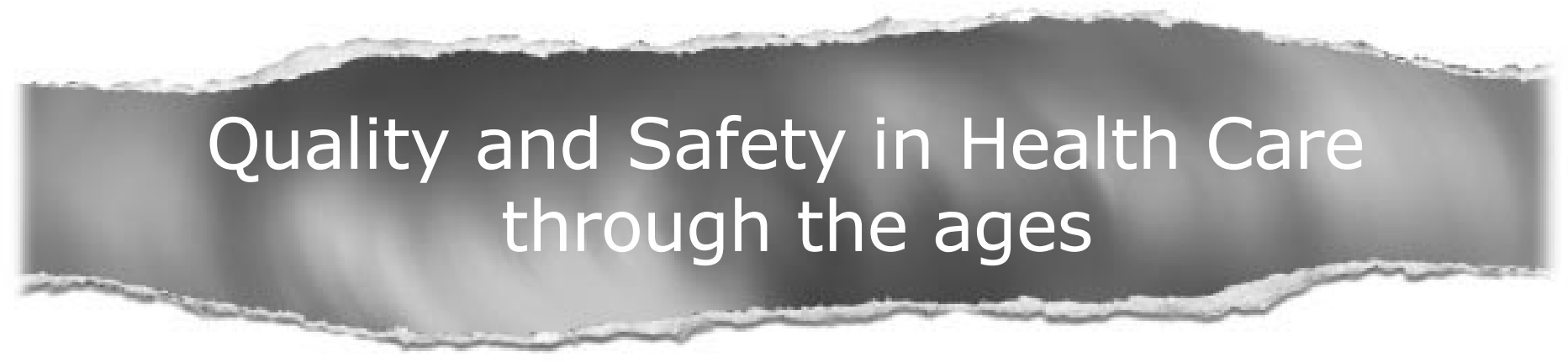

Browse the Archive

Quality and Safety in Health Care online has an archive of content dating back to 1992.

Full text from January 2000; abstracts from 1992; table of contents from 1992

www.qualityhealthcare.com 